\begin{tabular}{|c|} 
Original \\
article
\end{tabular}

\title{
Periurethral gland abscess: aetiology and treatment
}

\author{
Cornelus J G Sanders, Martina M M Mulder
}

Objectives: To establish some characteristics of patients with periurethral gland abscess, its microbiological profile, and response to treatment.

Method: The patients were seen at the Khami Road Clinic, Bulawayo, a municipal STD referral clinic, serving an urban population. Twenty consecutive men with periurethral abscesses were studied. Demographic data and a sexual history were obtained from each patient. Aspirates from the abscess cavities and urethral swabs were collected for microbiology, and blood samples taken for syphilis and HIV serology. The patients were treated by aspiration of the abscess cavities, followed by a single injection of kanamycin $2.0 \mathrm{~g}$ followed by a 1 week course of oral doxycycline $100 \mathrm{mg}$ twice daily.

Results: Neisseria gonorrhoeae was cultured from three aspirates and five urethral specimens. Chlamydia trachomatis was found in two aspirates and three urethral specimens. Other organisms isolated included Gram negative and anaerobic bacilli. HIV antibody was detected in 13 of 18 patients tested. The response to initial treatment was good, but the abscesses ruptured in two patients, one of whom developed a urinary fistula. One patient required treatment with an alternative antimicrobial regimen.

Conclusion: This study demonstrated a role for $N$ gonorrhoeae and possibly for $C$ trachomatis in the aetiology of periurethral abscess. The prevalence of HIV infection in these patients was high. The results of treatment of periurethral abscess by aspiration of pus and followed by antimicrobial therapy covering both $N$ gonorrhoeae and $C$ trachomatis were acceptable.

(Sex Transm Inf 1998;74:276-278)

Keywords: periurethral abscess; STDs; HIV infection

\section{Introduction}

Urethritis is a common problem in men in Africa, ${ }^{1}$ the most frequent causes being Neisseria gonorrhoeae and Chlamydia trachomatis. The exact incidence of these infections is not known but a recent study in a general population in Tanzania indicated a prevalence of $2.2 \%$ and $0.7 \%$ respectively. ${ }^{1}$ Complications such as lymphangitis and epididymo-orchitis, and facilitation of the transmission of HIV, are well recognised. ${ }^{2}$ Other complications such as urethral stricture and periurethral abscess occur less often in the antibiotic era. ${ }^{3}$ However, in our setting we see periurethral abscesses regularly, although their exact incidence is not known (fig 1).

Risk factors for periurethral abscess are urethritis, obstruction (for example, urethral stricture), and trauma, sometimes related to instrumentation of the urethra. ${ }^{3}$ Rarely, a urethral carcinoma may be implicated in the pathogenesis. ${ }^{34}$ In cultures from these abscesses anaerobic and Gram negative bacteria are commonly found. ${ }^{5}$ Periurethral abscesses may be complicated by urethral fistula, stricture, and necrotising fasciitis.

This study was undertaken to establish demographic and historical characteristics of patients presenting to our clinic with periurethral abscesses who had not received previous antibiotic treatment. We also established a microbiological profile of abscess aspirates and urethral specimens, and monitored the response of the patients to treatment. The patients were screened for HIV and syphilis.

\section{Patients and methods}

The study was conducted from May until December 1996 at the STD referral clinic (Khami Road Clinic) of Bulawayo City. This clinic serves a mainly urban population. Three patients included in this study had been referred from the surrounding provinces.

Twenty consecutive patients with a clinical diagnosis of periurethral abscess with or without concomitant urethral discharge were asked to participate in the study. They had not received treatment elsewhere. Oral informed consent to participate in the study was obtained, and a questionnaire on demographic data and sexual history completed for each patient. The following details were collected: age, marital status, history of previous STD, recent sexual history, and symptoms and their duration.

The skin overlying the abscesses was cleaned with chlorhexidine $1 \%$ solution and $70 \%$ alcohol and allowed to dry. The pus was aspirated through a fine needle into a sterile syringe, and inoculated into the following media: New York City medium for $N$ gonorrhoeae, MacConkey agar for aerobic organisms, blood agar for anaerobic bacteria, and chocolate agar for fastidious aerobic and facultative anaerobic organisms. All plates were incubated within 1 hour of inoculation. For the detection of $C$ trachomatis, a specimen of pus was placed in a cryotube of transport medium, which was stored at $4^{\circ} \mathrm{C}$ until transported to the laboratory, where a chlamydia 


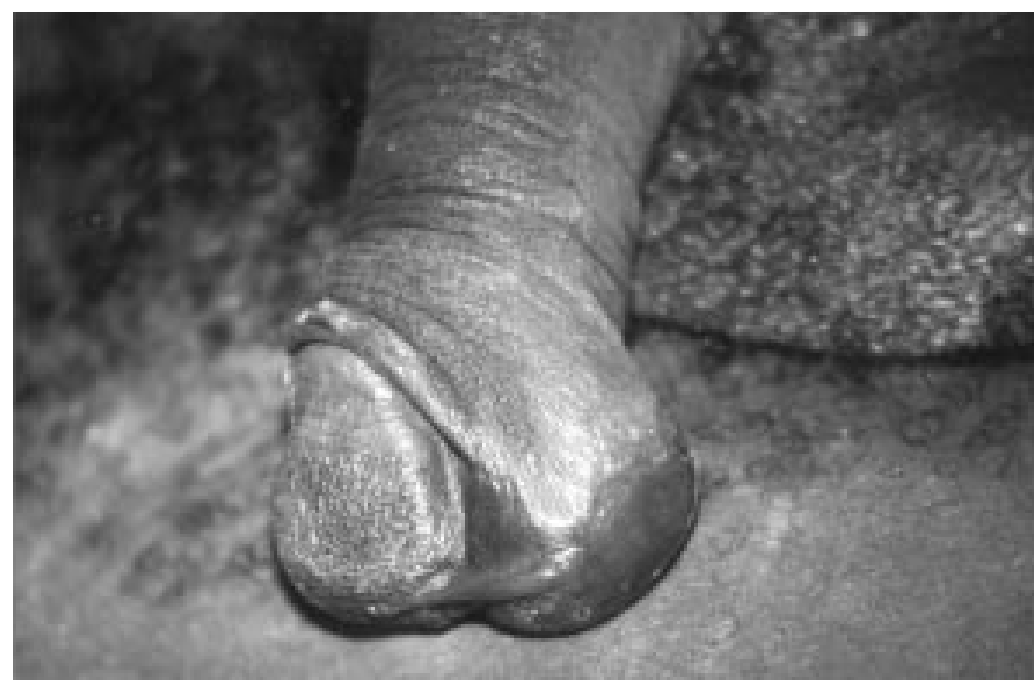

Figure 1 Periurethral gland abscess.

EIA test (Chlamydiazyme, Abbott) was performed. Urethral and endourethral swabs were taken for the identification of $N$ gonorrhoeae and $C$ trachomatis. Urinalysis was not performed. Serological tests for syphilis were a rapid plasma reagin test (RPR, SA Biokitt), positive results being confirmed by TPHA (Fujirebio). Patients were offered an HIV antibody test after pretest counselling; two patients refused. Serum specimens were tested with HIV-1/HIV-2, 3rd Generation Plus EIA (Abbott Laboratories) and a positive test result confirmed by western blot (Biorad).

Treatment consisted of complete aspiration of pus from the abscess cavity followed by kanamycin $2 \mathrm{~g}$ intramuscularly immediately, then doxycycline $100 \mathrm{mg}$ twice daily for 1 week. This is the recommended syndromic treatment for urethritis in Zimbabwe, adapted from guidelines of the World Health Organisation. ${ }^{5}$ The patients were asked to return after 1 week to assess the response to treatment, or earlier if any adverse effects occurred.

\section{Results}

The majority of periurethral abscesses encountered were localised along the pars spongiosa and bulbar part of the urethra. One patient had an abscess thought to originate from Cowper's glands. The mean age of patients was 30.4 years (range $23-41$ years); 13 were married and seven single. None of the patients gave a history of periurethral abscess or instrumentation of the urethra. Fifteen patients described previous episodes of genital infection-seven had had genital ulcer, five urethral discharge, and three both conditions. A recent history of dysuria and/or urethral discharge was given by 11 patients, with a mean duration of 10.3 days, but in only six of these men was a discharge noted on examination.

The results of laboratory examination of abscess aspirates and urethral specimens are given in table 1. $N$ gonorrhoeae was recovered from three abscess aspirates and from five urethral swabs, and $C$ trachomatis from two aspirates and three swabs. Other microbes isolated included Gram negative organisms such as $E$ coli, Enterobacter spp, and anaerobes.
Table 1 Microbiological examination of abscess aspirates and urethral specimen

\begin{tabular}{ll}
\hline Abscess aspirates: & \\
Escherichia coli & 5 \\
Neisseria gonorrhoeae & 3 \\
Bacteroides species & 2 \\
Chlamydia trachomatis & 2 \\
Enterobacter spp & 2 \\
Proteus mirabilis & 1 \\
Staphylococcus epidermidis & 1 \\
Urethral specimen: & \\
Neisseria gonorrhoeae & 5 \\
Chlamydia trachomatis & 3 \\
\hline
\end{tabular}

Three men gave positive RPR reactions, confirmed by TPHA in two, and HIV antibody was detected in 13 of the 18 patients tested (72.2\%).

Four patients were lost to follow up. Of the remaining 16, two returned after 1 and 3 days respectively because of a spontaneous abscess rupture. One of these had symptoms of a urinary fistula and was referred for further management. The other patient received debridement of the abscess area and continued treatment. On follow up, this and 13 other patients showed healing or healed abscesses without clinical signs of fistulas or urinary tract obstruction. One patient showed no improvement and was treated with ceftriaxone and erythromycin after which resolution of symptoms occurred.

\section{Discussion}

The male urethra is lined with stratified or pseudostratified columnar epithelium. This type of epithelium is readily infected by $N$ gonorrhoeae and $C$ trachomatis. ${ }^{2}{ }^{6}$ Subepithelial progression of an infection may lead to periurethritis, infection of the periurethral glands, and abscess formation. Predisposing factors to this event include a history of gonorrhoea, previous abscesses, and urethral stricture. ${ }^{5}$ If the abscess penetrates Buck's fascia a necrotising fasciitis may may occur, with extensive tissue destruction.

The mean age of our patients (30.4 years) was younger than the average age ( 56.7 years) reported by Walther et al. ${ }^{3}$ This may partially explain the relative high number of sexually transmitted pathogens that were isolated in our study. A comparable result has been reported in epididymitis, in which urinary pathogens are found more frequently in older men. ${ }^{7}$

Eight patients gave a previous history of urethral discharge. This supports the idea that men with previous inflammatory changes or possible strictures of the urethra are more likely to develop a periurethral abscess with subsequent infections. ${ }^{3}$

It has been postulated that the pathogenesis of periurethral abscess may involve a urethral stricture with subsequent urethral disruption and extravasation of infected urine. ${ }^{3}$ Several organisms commonly found in urinary tract infections were encountered in our study. There have been few reports of organisms recovered from men with urethral abscesses. We detected $N$ gonorrhoeae and $C$ trachomatis in three and two aspirates respectively. Although the Chlamydiazyme test is not validated for the detection of $C$ trachomatis in pus, it is possible 
that these organisms are involved in the pathogenesis of periurethral abscess. HIV antibody was detected in 13 of the 18 patients tested (72.2\%). In 1995, 73.3\% HIV seropositivity rate was found in the HIV sentinel serosurvey among STD patients in Bulawayo City. ${ }^{8}$ We cannot say whether the risk of periurethral abscess is increased in the presence of HIV infection or if these abscesses may facilitate HIV transmission. We found clinical evidence of AIDS in three patients with periurethral abscess, all because of concurrent Mycobacterium tuberculosis infection. Others had minor skin abnormalities or lymphadenopathy, and seven patients were asymptomatic.

In industrialised countries treatment of periurethral abscess by wide debridement and immediate suprapubic urinary diversion has been advocated. ${ }^{3}$ In our setting the response to syndromic treatment for urethral discharge together with aspiration of pus appeared to be reasonably good. Serious sequelae were encountered in one patient, who developed a urinary tract fistula. Another patient, in whom spontaneous rupture of his abscess occurred, continued treatment and when reviewed had improved clinically. A third patient required treatment with different antimicrobials after an initial poor response. No other complications were seen during the admittedly short follow up periods.

Contributors: Dr Sanders was the main investigator who designed the study, conducted patient management, collected and analysed the data, and wrote the manuscript. Dr Mulder participated in patient management and writing the manuscript.

1 Grosskurth H, Mayaud P, Mosha F, et al. Asymptomatic gonorrhoea and chlamydial infection in rural Tanzanian men. BMF 1996;312:277-80.

2 Hook III EW, Handsfield HH. Gonococcal infections in the adult. In: Holmes KK, Mårdh P-A, Sparling PF, Wiesner adult. In: Holmes KK, Mardh P-A, Sparling PF, Wiesner PJ, eds. Sexually transmitted

3 Walther MM, Mann BB, Finnerty DP. Periurethral abscess. F Urol 1987;138:1167-70

Angulo JC, Larrinaga JR, Unda-Urzaiz M, et al. Squamous cell carcinoma of the male urethra mimicking a paravertebral abscess. Urol Int 1992;48:108-10.

5 World Health Organisation. Management of sexually transmitted diseases. Geneva: WHO/GPA, 1994 (WHO/GPA TEM/94.1).

6 Stamm WE, Holmes KK. Chlamydia trachomatis infections of the adult. In: Holmes KK, Mårdh P-A, Sparling PF, Wiesner PJ, eds. Sexually transmitted diseases. 2nd ed. New York: McGraw-Hill, 1990:181-93.

7 Hoosen AA, O'Farrell N, Ende van den J. Microbiology of acute epididymitis in a developing community. Genitourin Med 1993;69:361-3.

8 HIV seroprevalence survey among STD patients, 1995. Bulawayo City: Health Services Department, 1995. 